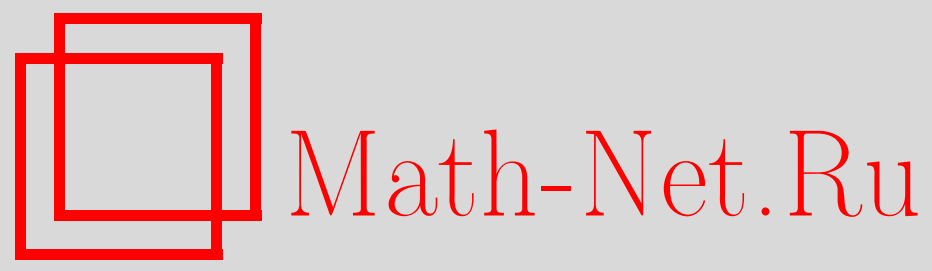

Н. С. Анофрикова, М. В. Вильде, Низкочастотные длинноволновые приближения трёхмерных динамических уравнений для случая двухслойной вязкоупругой пластины, Вестн. Сам. гос. техн. ун-та. Сер. Физ.мат. науки, 2012, выпуск 4(), 115-121

DOI: https://doi.org/10.14498/vsgtu1085

Использование Общероссийского математического портала Math-Net.Ru подразумевает, что вы прочитали и согласны с пользовательским соглашением

http://www.mathnet.ru/rus/agreement

Параметры загрузки:

IP: 18.209 .158 .208

26 апреля 2023 г., 17:11:36

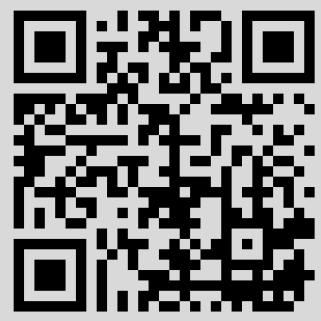




\title{
НИЗКОЧАСТОТНЫЕ ДЛИННОВОЛНОВЫЕ ПРИБЛИЖЕНИЯ ТРЁХМЕРНЫХ ДИНАМИЧЕСКИХ УРАВНЕНИЙ ДЛЯ СЛУЧАЯ ДВУХСЛОЙНОЙ ВЯЗКОУПРУГОЙ ПЛАСТИНЫ
}

\author{
Н. С. Анофрикова, М.В. Вилъде \\ Саратовский государственный университет им. Н. Г. Чернышевского, \\ 410012, Россия, Саратов, ул. Астраханская, 83. \\ E-mails: nanofrikova@yandex.ru, mv_wilde@mail.ru
}

\begin{abstract}
Представлены асимптотические методы, разработанные для вывода низкочастотных длинноволновых приближений трёхмерных динамических уравнений для случая двухслойной пластины, выполненной из вязкоупругих материалов. Путём асимптотического интегрирования точных трёхмерных уравнений выведены двумерные уравнения для асимптотически главных компонент напряжённо-деформированного состояния для тангенииального и поперечного приближений.
\end{abstract}

Ключевые слова: двухслойная пластина, вязкоупругость, асимптотические методы, низкочастотные длинноволновые приближения.

Введение. В работах [1-5] описаны асимптотические методы, разработанные для исследования динамического поведения тонкостенных конструкций различной геометрии, выполненных из упругих и вязкоупругих материалов. В [1] рассмотрены асимптотические приближения трёхмерных уравнений теории упругости для тонких пластин и оболочек. Описаны четыре типа приближений, выделенные в зависимости от значений показателей изменяемости и динамичности, а также представлено использование этих приближений для решения динамических задач различных типов, в частности, нестационарных задач для оболочек вращения. Асимптотический подход к решению задач подобного класса описан также в монографии [2]. В работе [3] систематизированы результаты разработки асимптотических методов исследования нестационарных волн для тонких упругих оболочек вращения. В [4] были решены аналогичные задачи для случая вязкоупругой оболочки. Статья [5] посвящена изложению методики построения низкочастотных длинноволновых приближений для случая двухслойной упругой пластины. В настоящей работе предлагается методика построения аналогичных приближений для случая двухслойной пластины, выполненной из вязкоупругих материалов.

1. Постановка задачи. Рассмотрим бесконечную двухслойную пластину, оба слоя которой выполнены из вязкоупругих материалов, свойства которых описываются моделью стандартного вязкоупругого тела с условием упругого объемного расширения. Введем декартову систему координат $\left(x_{1}, x_{2}, z\right)$, совмещая плоскость $O x_{1} x_{2}$ со срединной плоскостью пластины и направляя ось $z$ по нормали к срединной плоскости. Введем обозначения: $l$ - номер слоя $(l=1,2), \sigma_{i j}^{(l)}$ - напряжения, $u_{i}^{(l)}$ - перемещения в $l$-том слое пластины; $2 h_{l}-$

Наталия Сергеевна Анофрикова (к.ф.-м.н., доц.), доцент, каф. математической теории упругости и биомеханики.

Мария Владимировна Вилъде (д.ф.-м.н.), ведущий научный сотрудник, образовательнонаучный институт наноструктур и биосистем. 
толщина $l$-того слоя, $2 h$ - толщина пластины.

Будем предполагать, что наружные поверхности пластины свободны от нагрузки. Тогда граничные условия на них имеют вид $(k=1,2,3)$ :

$$
\begin{array}{rrr}
\text { при } & z=-h: & \sigma_{3 k}^{(2)}=0, \\
\text { при } & z=h: & \sigma_{3 k}^{(1)}=0 .
\end{array}
$$

Граничные условия на стыке двух слоев пластины - условия непрерывного контакта - сформулируем следующим образом:

$$
\text { при } \quad z=z_{1}: \quad \sigma_{3 k}^{(1)}=\sigma_{3 k}^{(2)}, \quad u_{k}^{(1)}=u_{k}^{(2)},
$$

где $z_{1}=h-2 h_{1}$.

Приведём точные трёхмерные динамические уравнения теории вязкоупругости для пластины. Уравнения движения возьмём в виде

$$
\frac{\partial \sigma_{i i}^{(l)}}{\partial x_{i}}+\frac{\partial \sigma_{j i}^{(l)}}{\partial x_{j}}+\frac{\partial \sigma_{3 i}^{(l)}}{\partial z}-\rho_{l} \frac{\partial^{2} u_{i}^{(l)}}{\partial t^{2}}=0, \quad \frac{\partial \sigma_{i 3}^{(l)}}{\partial x_{i}}+\frac{\partial \sigma_{j 3}^{(l)}}{\partial x_{j}}+\frac{\partial \sigma_{33}^{(l)}}{\partial z}-\rho_{l} \frac{\partial^{2} u_{3}^{(l)}}{\partial t^{2}}=0
$$

где $\rho_{l}$ - плотность материала слоя, $t-$ время.

Исходя из уравнений, приведённых в [6], уравнения состояния для $l$-того слоя можно записать следующим образом:

$$
\begin{aligned}
& E_{l}\left(\frac{1}{t_{2 l}}+\frac{\partial}{\partial t}\right) \frac{\partial u_{i}^{(l)}}{\partial x_{i}}=\left[\frac{1}{3}\left(\frac{1-2 \nu_{l}}{t_{2 l}}+2 \frac{1+\nu_{l}}{t_{1 l}}\right)+\frac{\partial}{\partial t}\right] \sigma_{i i}^{(l)}+ \\
& +\left[\frac{1}{3}\left(\frac{1-2 \nu_{l}}{t_{2 l}}-\frac{1+\nu_{l}}{t_{1 l}}\right)-\nu_{l} \frac{\partial}{\partial t}\right]\left(\sigma_{j j}^{(l)}+\sigma_{33}^{(l)}\right) \\
& \begin{aligned}
E_{l}\left(\frac{1}{t_{2 l}}+\frac{\partial}{\partial t}\right) \frac{\partial u_{3}^{(l)}}{\partial z}=\left[\frac{1}{3}\right. & \left.\left(\frac{1-2 \nu_{l}}{t_{2 l}}+2 \frac{1+\nu_{l}}{t_{1 l}}\right)+\frac{\partial}{\partial t}\right] \sigma_{33}^{(l)}+ \\
& +\left[\frac{1}{3}\left(\frac{1-2 \nu_{l}}{t_{2 l}}-\frac{1+\nu_{l}}{t_{1 l}}\right)-\nu_{l} \frac{\partial}{\partial t}\right]\left(\sigma_{i i}^{(l)}+\sigma_{j j}^{(l)}\right),
\end{aligned} \\
& \frac{E_{l}}{1+\nu_{l}}\left(\frac{1}{t_{2 l}}+\frac{\partial}{\partial t}\right)\left(\frac{\partial u_{i}^{(l)}}{\partial x_{j}}+\frac{\partial u_{j}^{(l)}}{\partial x_{i}}\right)=2\left(\frac{1}{t_{1 l}}+\frac{\partial}{\partial t}\right) \sigma_{i j}^{(l)}, \\
& \frac{E_{l}}{1+\nu_{l}}\left(\frac{1}{t_{2 l}}+\frac{\partial}{\partial t}\right)\left(\frac{\partial u_{i}^{(l)}}{\partial z}+\frac{\partial u_{3}^{(l)}}{\partial x_{i}}\right)=2\left(\frac{1}{t_{1 l}}+\frac{\partial}{\partial t}\right) \sigma_{3 i}^{(l)}(i \neq j=1,2 ; l=1,2),
\end{aligned}
$$

где $t_{1 l}$ - характерное время релаксации, $t_{2 l}$ - характерное время ползучести, $E_{l}, \nu_{l}$ - мгновенные значения модуля Юнга и коэффициент Пуассона материала $l$-того слоя соответственно.

Произведём в уравнениях (3), (4) растяжение масштабов независимых переменных по формулам

$$
x_{i}=L \eta^{q} \xi_{i}, \quad z=L \eta \zeta, \quad t=L c_{21}^{-1} \eta^{a} \tau,
$$


где $q$ - показатель изменяемости, $a$ - показатель динамичности, $c_{21}-$ скорость волны сдвига в первом слое, $\eta=h L^{-1} \ll 1$-относительная полутолщина пластины, $L$ - характерный размер длины.

Предположим, что дифференцирование по безразмерным переменным $\xi_{i}$, $\zeta, \tau$ не меняет асимптотический порядок неизвестных величин. Кроме того, будем рассматривать случай, когда скорости волн сдвига для материалов первого и второго слоев - величины одного порядка. Введение независимых переменных (5) позволяет методом асимптотического интегрирования трёхмерных уравнений теории вязкоупругости (3), (4) вывести асимптотически приближенные уравнения для составляющих напряжённо-деформированного состояния (НДС) при различных показателях изменяемости и динамичности.

В настоящей работе остановимся на случае так называемых длинноволновых низкочастотных приближений. K этому виду относятся приближения, для которых показатели динамичности и изменяемости удовлетворяют неравенствам $q<1, a<1$ [2]. Длинноволновые приближения разделяют на тангенциальные и поперечные, соответствующие теориям растяжения и изгиба тонких пластин соответственно.

2. Низкочастотные длинноволновые тангенциальные приближения. Начнём рассмотрение с тангенциального приближения. В этом случае тангенциальные компоненты вектора перемещений велики по сравнению с его нормальной компонентой: $u_{i}^{(l)} \gg u_{3}^{(l)}(i=1,2)$.

Оценим величины времен ползучести и релаксации, вводя показатели их интенсивности по формулам

$$
t_{i l}=L c_{21}^{-1} \eta^{r_{i l}} \tau_{i l}
$$

и будем предполагать, что $r_{i l} \leqslant a(i=1,2)$.

При построении тангенциального приближения показатели изменяемости и динамичности для каждого слоя связаны соотношением $q=a$. Введем следующие асимптотики для компонент НДС [5]:

$$
\begin{gathered}
u_{i}^{(l)}=L \eta^{q} \tilde{u}_{i}^{(l)}, \quad u_{3}^{(l)}=L \eta \tilde{u}_{3}^{(l)}, \quad \sigma_{i i}^{(l)}=E_{l} \tilde{\sigma}_{i i}^{(l)}, \quad \sigma_{i j}^{(l)}=E_{l} \tilde{\sigma}_{i j}^{(l)}, \\
\sigma_{3 i}^{(l)}=E_{l} \eta^{1-q} \tilde{\sigma}_{3 i}^{(l)}, \quad \sigma_{33}^{(l)}=E_{l} \eta^{2-2 q} \tilde{\sigma}_{33}^{(l)} \quad(i, j=1,2) .
\end{gathered}
$$

Предполагаем, что величины с «тильдой» имеют один и тот же асимптотический порядок.

В силу выбора асимптотик (7), в уравнения движения, записанные с учётом $(5),(6)$, в рамках погрешности $O\left(\eta^{2-2 q}\right)$ входят слагаемые, содержащие производные по $\zeta$ от $\tilde{\sigma}_{3 i}^{(l)}(i=1,2,3)$. Такой выбор асимптотик позволяет удовлетворить всем граничным условиям при асимптотическом интегрировании.

В результате асимптотического интегрирования уравнений $(3),(4)$ устанавливаем следующую зависимость компонент НДС от нормальной координаты:

$$
\begin{gathered}
\tilde{u}_{i}^{(l)}=u_{i, 0}^{(l)}, \quad \tilde{u}_{3}^{(l)}=u_{3,0}^{(l)}+\zeta u_{3,1}^{(l)}, \quad \tilde{\sigma}_{i i}^{(l)}=\sigma_{i i, 0}^{(l)}, \quad \tilde{\sigma}_{i j}^{(l)}=\sigma_{i j, 0}^{(l)}, \\
\tilde{\sigma}_{3 i}^{(l)}=\sigma_{3 i, 0}^{(l)}+\zeta \sigma_{3 i, 1}^{(l)}, \quad \tilde{\sigma}_{33}^{(l)}=\sigma_{33,0}^{(l)}+\zeta \sigma_{33,1}^{(l)}+\zeta^{2} \sigma_{33,2}^{(l)},
\end{gathered}
$$

где величины с цифрой после запятой в нижнем индексе от $\zeta$ не зависят. 
Переход в граничных условиях (1), (2) к представлениям (7), (8) позволяет установить связь между компонентами НДС первого и второго слоёв:

$$
u_{i, 0}^{(1)}=u_{i, 0}^{(2)}, \quad 2 E_{1} h_{1} \sigma_{3 i, 1}^{(1)}+2 E_{2} h_{2} \sigma_{3 i, 1}^{(2)}=0 .
$$

В результате асимптотического интегрирования получены: система относительно асимптотически главных компонент НДС $u_{i, 0}^{(l)}, \sigma_{i i, 0}^{(l)}, \sigma_{i j, 0}^{(l)}$ и система, определяющая асимптотически второстепенные компоненты через асимптотически главные.

Приведём вид размерной двумерной формы записи полученной системы для асимптотически главных компонент НДС. Введём перемещения $u_{i}$, усилия $T_{i}, S_{i j}$ и усреднённую плотность $\rho$ по формулам

$$
\begin{gathered}
u_{i}=L \eta^{q} u_{i}^{(1)}=L \eta^{q} u_{i}^{(2)}, \quad T_{i}=2\left(h_{1} \sigma_{i i}^{(1)}+h_{2} \sigma_{i i}^{(2)}\right), \\
S_{i j}=2\left(h_{1} \sigma_{i j}^{(1)}+h_{2} \sigma_{i j}^{(2)}\right), \quad \rho=\left(\rho_{1} h_{1}+\rho_{2} h_{2}\right) / h .
\end{gathered}
$$

С учётом (9) система разрешающих уравнений для асимптотически главных компонент НДС примет вид

$$
\begin{aligned}
& \frac{\partial T_{i}}{\partial x_{i}}+\frac{\partial S_{i j}}{\partial x_{j}}-2 \rho h \frac{\partial^{2} u_{i}}{\partial t^{2}}=0 \\
& {\left[\frac{h_{1} E_{1}}{1+\nu_{1}} f_{12} f_{21}+\frac{h_{2} E_{2}}{1+\nu_{2}} f_{11} f_{22}\right]\left(\frac{\partial u_{i}}{\partial x_{j}}+\frac{\partial u_{j}}{\partial x_{i}}\right)=f_{11} f_{12} S_{i j},} \\
& 2\left[h_{1} E_{1} f_{21} f_{31}\left(f_{32}^{2}-f_{42}^{2}\right)+h_{2} E_{2} f_{22} f_{32}\left(f_{31}^{2}-f_{41}^{2}\right)\right] \frac{\partial u_{i}}{\partial x_{i}}- \\
& \quad-2\left[h_{1} E_{1} f_{21} f_{41}\left(f_{32}^{2}-f_{42}^{2}\right)+h_{2} E_{2} f_{22} f_{42}\left(f_{31}^{2}-f_{41}^{2}\right)\right] \frac{\partial u_{j}}{\partial x_{j}}= \\
& \quad=\left(f_{31}^{2}-f_{41}^{2}\right)\left(f_{32}^{2}-f_{42}^{2}\right) T_{i},
\end{aligned}
$$

где

$$
\begin{gathered}
f_{i l}=\left(\frac{1}{t_{i l}}+\frac{\partial}{\partial t}\right), \quad f_{3 l}=\frac{1}{3}\left(\frac{1-2 \nu_{l}}{t_{2 l}}+2 \frac{1+\nu_{l}}{t_{1 l}}\right)+\frac{\partial}{\partial t}, \\
f_{4 l}=\frac{1}{3}\left(\frac{1-2 \nu_{l}}{t_{2 l}}-\frac{1+\nu_{l}}{t_{1 l}}\right)-\nu_{l} \frac{\partial}{\partial t}, \quad i \neq j=1,2 ; l=1,2 .
\end{gathered}
$$

3. Низкочастотное длинноволновое поперечное приближение. Теперь рассмотрим случай поперечного приближения. В этом случае нормальная компонента вектора перемещений велика по сравнению с его тангенциальными компонентами: $u_{3}^{(l)} \gg u_{i}^{(l)}(i=1,2)$.

Величины времен ползучести и релаксации будем оценивать так же, как и в предыдущем случае, вводя показатели их интенсивности по формулам (6). Как и раньше, будем предполагать, что $r_{i l} \leqslant a(i=1,2)$.

При построении поперечного приближения показатели изменяемости и динамичности для каждого слоя связаны соотношением $q=2 a-1, q>1 / 2$. Введём следующие асимптотики для компонент НДС:

$$
\begin{gathered}
u_{i}^{(l)}=L \eta \tilde{u}_{i}^{(l)}, \quad u_{3}^{(l)}=L \eta^{q} \tilde{u}_{3}^{(l)}, \quad \sigma_{i i}^{(l)}=E_{l} \eta^{1-q} \tilde{\sigma}_{i i}^{(l)}, \quad \sigma_{i j}^{(l)}=E_{l} \eta^{1-q} \tilde{\sigma}_{i j}^{(l)}, \\
\sigma_{3 i}^{(l)}=E_{l} \eta^{2-2 q} \tilde{\sigma}_{3 i}^{(l)}, \quad \sigma_{33}^{(l)}=E_{l} \eta^{3-3 q} \tilde{\sigma}_{33}^{(l)} \quad(i, j=1,2) .
\end{gathered}
$$


Предполагается, что величины с «тильдой» имеют один и тот же асимптотический порядок.

Как и в случае тангенциального приближения, в силу выбора асимптотик (11) в уравнения движения, записанные с учётом (5), (6), в рамках погрешности $O\left(\eta^{2-2 q}\right)$ входят слагаемые, содержащие производные по $\zeta$ от $\tilde{\sigma}_{3 i}^{(l)}(i=$ $=1,2,3)$. Такой выбор асимптотик позволяет удовлетворить всем граничным условиям при асимптотическом интегрировании.

Зависимость компонент НДС от нормальной координаты, установленная в результате асимптотического интегрирования уравнений $(3),(4)$, в данном случае имеет вид

$$
\begin{array}{ll}
\tilde{u}_{i}^{(l)}=u_{i, 0}^{(l)}+\zeta u_{i, 1}^{(l)}, \quad \tilde{u}_{3}^{(l)}=u_{3,0}^{(l)}, & \tilde{\sigma}_{i i}^{(l)}=\sigma_{i i, 0}^{(l)}+\zeta \sigma_{i i, 1}^{(l)}, \quad \tilde{\sigma}_{i j}^{(l)}=\sigma_{i j, 0}^{(l)}+\zeta \sigma_{i j, 1}^{(l)}, \\
\tilde{\sigma}_{3 i}^{(l)}=\sigma_{3 i, 0}^{(l)}+\zeta \sigma_{3 i, 1}^{(l)}+\zeta^{2} \sigma_{3 i, 2}^{(l)}, & \tilde{\sigma}_{33}^{(l)}=\sigma_{33,0}^{(l)}+\zeta \sigma_{33,1}^{(l)}+\zeta^{2} \sigma_{33,2}^{(l)}+\zeta^{3} \sigma_{33,3}^{(l)},
\end{array}
$$

где величины с цифрой после запятой в нижнем индексе от $\zeta$ не зависят.

Переходя в граничных условиях (1), (2) к представлениям (11), (12), получим соотношения

$$
u_{i, 0}^{(1)}=u_{i, 0}^{(2)}, \quad u_{3,0}^{(1)}=u_{3,0}^{(2)} .
$$

Приведём вид двумерной разрешающей системы, полученной в результате асимптотического интегрирования системы (3), (4):

$$
\begin{aligned}
& \frac{\partial T_{i}}{\partial x_{i}}+\frac{\partial S_{i j}}{\partial x_{j}}=0, \quad \frac{\partial M_{i}}{\partial x_{i}}+\frac{\partial H_{i j}}{\partial x_{j}}-N_{i}=0, \quad \frac{\partial N_{1}}{\partial x_{1}}+\frac{\partial N_{2}}{\partial x_{2}}-2 \rho h \frac{\partial^{2} w}{\partial t^{2}}=0, \\
& {\left[\frac{h_{1} E_{1}}{1+\nu_{1}} f_{12} f_{21}+\frac{h_{2} E_{2}}{1+\nu_{2}} f_{11} f_{22}\right]\left(\frac{\partial u_{i}}{\partial x_{j}}+\frac{\partial u_{j}}{\partial x_{i}}\right)-} \\
& -2 h_{1} h_{2}\left[\frac{E_{1}}{1+\nu_{1}} f_{21} f_{12}-\frac{E_{2}}{1+\nu_{2}} f_{11} f_{22}\right] \frac{\partial^{2} w}{\partial x_{i} \partial x_{j}}=f_{11} f_{12} S_{i j}, \\
& 2\left[h_{1} E_{1} f_{21} f_{31}\left(f_{32}^{2}-f_{42}^{2}\right)+h_{2} E_{2} f_{22} f_{32}\left(f_{31}^{2}-f_{41}^{2}\right)\right] \frac{\partial u_{i}}{\partial x_{i}}- \\
& -2\left[h_{1} E_{1} f_{21} f_{41}\left(f_{32}^{2}-f_{42}^{2}\right)+h_{2} E_{2} f_{22} f_{42}\left(f_{31}^{2}-f_{41}^{2}\right)\right] \frac{\partial u_{j}}{\partial x_{j}}- \\
& -2 h_{1} h_{2}\left[E_{1} f_{21} f_{31}\left(f_{32}^{2}-f_{42}^{2}\right)-E_{2} f_{22} f_{32}\left(f_{31}^{2}-f_{41}^{2}\right)\right] \frac{\partial^{2} w}{\partial x_{i}^{2}}+ \\
& +2 h_{1} h_{2}\left[E_{1} f_{21} f_{41}\left(f_{32}^{2}-f_{42}^{2}\right)-E_{2} f_{22} f_{42}\left(f_{31}^{2}-f_{41}^{2}\right)\right] \frac{\partial^{2} w}{\partial x_{j}^{2}}= \\
& =\left(f_{31}^{2}-f_{41}^{2}\right)\left(f_{32}^{2}-f_{42}^{2}\right) T_{i}, \\
& -2 h_{1} h_{2}\left[E_{1} f_{21} f_{41}\left(f_{32}^{2}-f_{42}^{2}\right)-E_{2} f_{22} f_{42}\left(f_{31}^{2}-f_{41}^{2}\right)\right] \frac{\partial u_{j}}{\partial x_{j}}- \\
& -2\left[\frac{h_{1}}{3}\left(h_{1}^{2}+3 h_{2}^{2}\right) E_{1} f_{21} f_{31}\left(f_{32}^{2}-f_{42}^{2}\right)+\right.
\end{aligned}
$$




$$
\begin{gathered}
\left.+\frac{h_{2}}{3}\left(h_{2}^{2}+3 h_{1}^{2}\right) E_{2} f_{22} f_{32}\left(f_{31}^{2}-f_{41}^{2}\right)\right] \frac{\partial^{2} w}{\partial x_{i}^{2}}+ \\
+2\left[\frac{h_{1}}{3}\left(h_{1}^{2}+3 h_{2}^{2}\right) E_{1} f_{21} f_{41}\left(f_{32}^{2}-f_{42}^{2}\right)+\right. \\
\left.+\frac{h_{2}}{3}\left(h_{2}^{2}+3 h_{1}^{2}\right) E_{2} f_{22} f_{42}\left(f_{31}^{2}-f_{41}^{2}\right)\right] \frac{\partial^{2} w}{\partial x_{j}^{2}}= \\
\left.h_{1} h_{2}\left[\frac{E_{1}}{1+\nu_{1}} f_{12} f_{21}-\frac{E_{2}}{1+\nu_{2}} f_{11} f_{22}\right]\left(\frac{\partial u_{i}}{\partial x_{j}}+\frac{\partial u_{j}}{\partial x_{i}}\right)-f_{31}^{2}-f_{41}^{2}\right)\left(f_{32}^{2}-f_{42}^{2}\right) M_{i}, \\
-\left[\frac{2 h_{1}}{3}\left(h_{1}^{2}+3 h_{2}^{2}\right) \frac{E_{1}}{1+\nu_{1}} f_{21} f_{12}+\frac{2 h_{2}}{3}\left(h_{2}^{2}+3 h_{1}^{2}\right) \frac{E_{2}}{1+\nu_{2}} f_{11} f_{22}\right] \frac{\partial^{2} w}{\partial x_{i} \partial x_{j}}=
\end{gathered}
$$

где перемещения $u_{i}, w$, усилия $T_{i}, S_{i j}$, моменты $M_{i}, H_{i j}$, перерезывающие силы $N_{i}$ определяются по формулам

$$
\begin{gathered}
u_{i}=L \eta u_{i, 0}^{(1)}=L \eta u_{i, 0}^{(2)}, \quad w=L \eta^{q} u_{3,0}^{(1)}=L \eta^{q} u_{3,0}^{(2)} \\
T_{i}=\int_{-h}^{z_{1}} \sigma_{i i}^{(2)} d z+\int_{z_{1}}^{h} \sigma_{i i}^{(1)} d z, \quad S_{i j}=\int_{-h}^{z_{1}} \sigma_{i j}^{(2)} d z+\int_{z_{1}}^{h} \sigma_{i j}^{(1)} d z \\
M_{i}=\int_{-h}^{z_{1}} z \sigma_{i i}^{(2)} d z+\int_{z_{1}}^{h} z \sigma_{i i}^{(1)} d z, \quad H_{i j}=\int_{-h}^{z_{1}} z \sigma_{i j}^{(2)} d z+\int_{z_{1}}^{h} z \sigma_{i j}^{(1)} d z \\
N_{i}=\int_{-h}^{z_{1}} \sigma_{3 i}^{(2)} d z+\int_{z_{1}}^{h} \sigma_{3 i}^{(1)} d z
\end{gathered}
$$

а остальные величины имеют тот же смысл, что и раньше.

Из двумерных уравнений динамической теории вязкоупругости для скорости продольной волны по двумерной теории получаем следующее выражение:

$$
c^{2}=\frac{1}{\rho_{1} h_{1}+\rho_{2} h_{2}} \sum_{l=1}^{2} \frac{E_{l} h_{l}}{1-\nu_{l}^{2}} .
$$

Переходя в уравнениях (10), (13) к пределу при $t_{1 l} \rightarrow \infty$ и $t_{2 l} \rightarrow \infty$, можно получить двумерные уравнения тангенциального и поперечного приближений для упругой двухслойной пластины. Если в последних положить $E_{1}=E_{2}$ и $\nu_{1}=\nu_{2}$, то уравнения тангенциального приближения переходят в уравнения обобщенного плоского напряженного состояния, а уравнения поперечного приближения - в уравнения теории изгиба пластин Кирхгофа.

Работа выполнена при поддержке РФФИ (проект № 11-01-00545).

\section{БИБЛИОГРАФИЧЕСКИЙ СПИСОК}

1. Коссович Л. Ю. Нестационарные задачи теории упругих тонких оболочек. Саратов: Саратовск. ун-т, 1986. 176 с. [Kossovich L. Yu. Transient problems of theory of thin elastic shells. Saratov: Saratov University, 1986. 176 pp.] 
2. Kaplunov Ju.D., Kossovich L. Yu., Nolde E. V. Dynamics of thin walled elastic bodies. SanDiego: Academic Press, 1998. x+226 pp.

3. Коссович Л. Ю. Асимптотические методы в динамике оболочек при ударных воздействиях // Изв. Сарат. ун-та. Нов. сер. Сер. Математика. Механика. Информатика, 2008. T. 8, № 2. C. 12-33. [Kossovich L. Yu. Asymptotic methods in dynamics of shells under shock loading // Izv. Saratov. Univ. Mat. Mekh. Inform., 2008. Vol. 8, no. 2. Pp. 12-33].

4. Бажанова Н. С., Коссович Л.Ю., Сухоловская М. С. Нестационарные волны в вязкоупругих оболочках: модель Максвелла// Изв. высш. учеб. завед. Сев.-Кавк. Регион. Ecmeств. науки, 2000. № 2. C. 17-24. [Bazhanova N. S., Kosovich L. Yu., Sukholovskay M. S. Non-stationary in waves viscoelastic shells: Maxwell's model // Izv. Vyssh. Uchebn. Zaved., Sev.-Kavk. Reg., Estestv. Nauki, 2000. no. 2. Pp. 17-24].

5. Коссович Л. Ю., Шевцова Ю. В. Асимптотические приближения трёхмерных динамических уравнений теории упругости в случае двухслойных пластин // Проблемъ прочности и пластичности, 2005. №67. С. 102-110. [Kossovich L. Yu.. Shevtsova Yu. V. Asymptotic approaches of three-dimentional dynamic equations of elasticity theory in the case of two-layered plates // Problemy prochnosti i plastichnosti, 2005. no.67. Pp. 102-110].

6. Новацкий B. Динамика сооружений. М.: Госстройиздат, 1963. 376 с. [Nowacki W. Dynamics of Constructions. Moscow: Gosstroyizdat, 1963. 376 pp.]

MSC: 74J30; 74K25, 74K20, 74H10

\section{LOW-FREQUENCY LONG-WAVE APPROXIMATIONS OF THE 3D DYNAMIC EQUATIONS FOR THE CASE OF DOUBLE-LAYERED VISCOELASTIC PLATE}

\section{N. S. Anofrikova, M. V. Wilde}

Saratov State University named after N. G. Chernyshevsky, 83, Astrakhanskaya st., Saratov, 410012, Russia.

E-mails: nanofrikova@yandex.ru, mv_wilde@mail.ru

The asymptotic methods developed to obtain low-frequency long-wave approximations of the 3D dynamic equations for the case of double-layered viscoelastic plate are described. The 2D equations for the leading tangential and transverse approximations of stressstrain state are derived. The method of asymptotic integration of exact $3 D$ equations is applied.

Key words: double-layered plate, viscoelasticity, asymptotic methods, low-frequency long-wave approximations.

Original article submitted 19/V/2012;

revision submitted 14/IX/2012.

Nataliya S. Anofrikova (Ph. D. (Phys. \& Math.)), Associate Professor, Dept. of Mathematical Theory of Elasticity and Biomechanics.

Mariya V. Wilde (Dr. Sci. (Phys. \& Math.)), Leading Research Scientist, Educational Research Institute of Nanostructures and Biosystems. 